\title{
Microscopic description of anisotropic low-density dipolar Bose gases in two dimensions
}

\author{
A. Macia, ${ }^{1}$ F. Mazzanti, ${ }^{1}$ J. Boronat, ${ }^{1}$ and R. E. Zillich $^{2}$ \\ ${ }^{1}$ Departament de Física i Enginyeria Nuclear, Campus Nord B4-B5, Universitat Politècnica de Catalunya, ES-08034 Barcelona, Spain \\ ${ }^{2}$ Institut für Theoretische Physik, Johannes-Kepler Universität, Altenbergerstr. 69, AT-4040 Linz, Austria
}

(Received 26 May 2011; published 19 September 2011)

\begin{abstract}
A microscopic description of the zero-energy two-body ground state and many-body static properties of anisotropic homogeneous gases of bosonic dipoles in two dimensions at low densities is presented and discussed. By changing the polarization angle with respect to the plane, we study the impact of the anisotropy, present in the dipole-dipole interaction, on the energy per particle, comparing the results with mean-field predictions. We restrict the analysis to the regime where the interaction is always repulsive, although the strength of the repulsion depends on the orientation with respect to the polarization field. We present a series expansion of the solution of the zero-energy two-body problem, which allows us to find the scattering length of the interaction and to build a suitable Jastrow factor that we use as a trial wave function for both a variational and diffusion Monte Carlo simulation of the infinite system. We find that the anisotropy has an almost negligible impact on the ground-state properties of the many-body system in the universal regime where the scattering length governs the physics of the system. We also show that scaling in the gas parameter persists in the dipolar case up to values where other isotropic interactions with the same scattering length yield different predictions.
\end{abstract}

DOI: 10.1103/PhysRevA.84.033625

PACS number(s): 03.75.Hh, 67.10.Hk, 67.10.Fj

\section{INTRODUCTION}

Quantum dipolar systems of bosons and fermions have gathered much experimental and theoretical attention in recent years. In 2005, Griesmaier and collaborators [1] on one side, and Stuhler and collaborators [2] on the other, reported on the first experimental realization of a Bose condensate of ${ }^{52} \mathrm{Cr}$, where the dipolar moment of the atoms is so large $\left(\sim 6 \mu_{B}\right)$ that the effect of the dipole-dipole interaction is comparable in strength to the van der Waals forces. More recently, new and exciting results have been achieved with polar molecules of rubidium and potassium $\left({ }^{40} \mathrm{~K}^{87} \mathrm{Rb}\right)$ [3], which have not been easy to create due to strong loss rates in the population induced by chemical reactions $[5,6]$. A promising route toward a molecular Bose-Einstein condensate is Feshbach association of $\mathrm{Rb}$ and $\mathrm{Cs}$, which are not reactive [4]. One of the major advantages of polar molecules is that the electric dipole moments are remarkably larger than in the magnetic case of ${ }^{52} \mathrm{Cr}$ and can be tuned by applying an external electric field. Systems of polar molecules have been speculated to present interesting applications ranging from the control of chemical reactions [7] to practical applications of quantum information theory [8].

From the theoretical point of view, dipolar systems present novel and interesting phenomena that make them particularly appealing. On the one hand, the anisotropic character of the dipole-dipole interaction introduces additional degrees of freedom compared with other condensed matter systems that can potentially enrich the phase diagram. On the other hand, the interaction decreases at large distances as $r^{-3}$ and becomes long ranged in three dimensions (3D), in contrast to typical van der Waals forces. In two dimensions (2D), though, the interaction is still short ranged, but at the border between both regimes.

The potential $V_{d}(\mathbf{r})$ describing how two dipoles with dipolar moments $\mathbf{p}_{1}$ and $\mathbf{p}_{2}$ interact is given by

$$
V_{d}(\mathbf{r})=\frac{C_{d d}}{4 \pi}\left[\frac{\hat{\mathbf{p}}_{1} \cdot \hat{\mathbf{p}}_{2}-3\left(\hat{\mathbf{p}}_{1} \cdot \hat{\mathbf{r}}\right)\left(\hat{\mathbf{p}}_{2} \cdot \hat{\mathbf{r}}\right)}{r^{3}}\right]
$$

with $\mathbf{r}$ the relative position vector between them and $C_{d d}$ the coupling constant defining the strength of the interaction. For permanent magnetic dipoles, $C_{d d}=\mu_{0} \mu^{2}$ where $\mu_{0}$ is the permeability of vacuum and $\mu$ is the permanent dipole moment of the atoms. Alternatively, the electric dipole moment can be induced by an electric field $\mathbf{E}$, and, in this case, the coupling constant is $C_{d d}=d^{2} / \epsilon_{0}$, where $d=\tilde{\alpha} E$ with $\tilde{\alpha}$ the static polarizability and $\epsilon_{0}$ the permittivity of vacuum. For a system of fully polarized dipoles in 2D as the ones considered here, $\mathbf{p}_{1}$ and $\mathbf{p}_{2}$ are parallel and define a fixed direction in space (see Fig. 1). In this case, $V_{d}(\mathbf{r})$ simplifies to

$$
V_{d}(\mathbf{r})=\frac{C_{d d}}{4 \pi}\left[\frac{1-3 \lambda^{2} \cos ^{2} \theta}{r^{3}}\right],
$$

where $\lambda=\sin \alpha, \alpha$ being the angle formed by the normal to the plane and the polarization field, which is tilted toward the $x$ axis. In this expression, $r$ and $\theta$ stand for the in-plane distance and polar angle, respectively. Notice that, in contrast to what happens in three dimensions, $\alpha$ is fixed in the fully polarized system and, thus, $\lambda \leqslant 1$ is a constant of the problem for a given $\alpha$.

One of the consequences of the form of the interaction is that it can be either attractive or repulsive depending on the orientation with respect to the polarization field. This fact alone triggers interesting discussions about the static and dynamic properties of dipolar systems. For instance, the formation of a density instability observed in the elementary excitation spectrum as the formation of a deep roton minimum has been widely discussed [9-11], a feature that is absent when the interaction is reduced to its purely isotropic limit in 2D [12] corresponding to $\lambda=0$ in Eq. (2). The presence of two- and/or many-body bound states in stacks of dipolar layers has also raised interesting questions [13]. Many other aspects, including scattering properties in 2D [14-16], pseudopotential treatments [17], or the impact of the anisotropy on the superfluid properties of a dipolar Bose gas [18], have gathered 


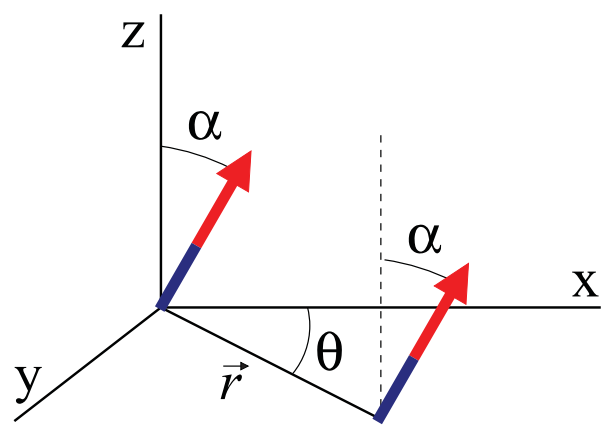

FIG. 1. (Color online) Two dipoles confined to move on the $X Y$ plane. The polarization field lays on the $X Z$ plane and fixes a direction in space forming an angle $\alpha$ with the $z$ axis.

interest in recent years. Most of these aspects and many others are covered in detail in the review article by Lahaye et al. [19]. In this paper, we concentrate on the case where the interaction is always repulsive, but is still anisotropic. That defines a critical angle $\alpha_{c}=0.615$ above which $V_{d}(\mathbf{r})$ starts to present negative contributions. We thus analyze the role of the anisotropy of the interaction in situations where the system is clearly prevented from collapse. Since $\alpha_{c}$ is reached at $\lambda^{2}=1 / 3$, we propose to use $\lambda^{2}$ as an expansion parameter.

Despite the relevance of the anisotropic character of the interaction in all these effects, not much effort has been put in the microscopic description of the wave function. The anisotropy makes the different angular momentum channels couple in a nontrivial way, and this introduces additional degrees of freedom that must be taken into account in a numerical simulation. At low densities, though, the problem simplifies since the zero-energy solution of the two-body scattering problem dominates the ground-state many-body wave function of the homogeneous gaseous phase. In this paper, we provide a detailed description of the zero-energy scattering wave function corresponding to two dipoles moving on the plane as a function of the polarization angle. Equipped with that solution, we build a microscopic variational manybody wave function that we use in a Monte Carlo simulation to describe the static properties of a gas of polarized dipoles in $2 \mathrm{D}$ at low densities.

The analysis of the low-density equation of state of a gas of weakly interacting particles has historically attracted great interest. Corrections to the mean-field prediction for three-dimensional [20] and one-dimensional [21] systems have been known for more than 50 years now. The two-dimensional case has been much more controversial as already the twobody problem presents logarithmic divergences in the leading scattering parameters that make series expansions difficult to carry out $[22,23]$. In any case, the low-density behavior of a gas of weakly interacting particles in 2D has been widely discussed in the literature for the case of isotropic interactions. One of the most remarkable properties exhibited by these systems is the universal behavior of the energy per particle, which admits a nonanalytic series expansion in the gas parameter $x=n a^{2}$, with $n$ the density and $a$ the $s$-wave scattering length. The leading-order, mean-field term in this series has been derived by several authors $[24,25]$ and reads as

$$
\epsilon_{m f}(x)=\left(\frac{2 m a^{2}}{\hbar^{2}}\right) \frac{E}{N}=\frac{4 \pi x}{|\ln x|} .
$$

The detailed form of the next-to-leading correction to this expression has been the subject of discussion and different authors proposed different forms in the past (see, for instance, Refs. [26,27]). The correct expression was recently derived in Ref. [28] and checked against numerically intense Monte Carlo simulations in Ref. [29]. For the model system of hard disks, the mean-field prediction of Eq. (3) holds well starting at $x \sim 0.001$ and down to quite low but still experimentally affordable values of the gas parameter [30]. However, no particular attention has been paid in all these works to the special case of anisotropic interactions.

In this paper, we discuss to what extent the mean-field law of Eq. (3) holds for the special case of the spatially anisotropic dipole-dipole interaction of Eq. (2) when the polarization angle varies between 0 and $\alpha_{c}$. We are particularly interested in discerning whether the angular dependence of the interaction has a noticeable impact on the mean-field prediction of Eq. (3) and on other relevant ground-state properties. In order to do that, we first solve in Sec. II the zero-energy two-body scattering problem and obtain an expression for the scattering length as a function of the polarization angle. We then use this result to build in Sec. III a variational many-body wave function of the Jastrow form that we use as an input to both a variational Monte Carlo (VMC) and a diffusion Monte Carlo (DMC) calculation, from which we obtain the equation of state as a function of $x$. For the sake of completeness, we also analyze the pair distribution function, the static structure factor, the one-body density matrix, and the condensate fraction, and discuss how these quantities scale on the gas parameter for different polarization angles and densities. Finally, in Sec. IV, the main conclusions of the work are summarized and discussed.

\section{ZERO-ENERGY TWO-BODY PROBLEM}

In this section, we develop a series expansion of the zero-energy scattering solution for two dipoles moving on the $X Y$ plane as a function of the polarization angle $\alpha$. This is done by first building the Green's function of the $\alpha=0$ case corresponding to a polarization field perpendicular to the plane. This is a particularly suitable situation since, in that case, the interaction is isotropic $[\lambda=0$ in Eq. (2)] and the Schrödinger equation can be exactly solved. From there, we carry out a series expansion in powers of $\lambda^{2}$ of the solution corresponding to the anisotropic case. The anisotropy makes the different angular momentum channels couple, and we report the expression for all orders in $\lambda^{2}$ contributing to each partial wave. We end this section by reporting the scattering length of the dipole-dipole interaction as a function of the polarization angle, required afterward to analyze the low-density properties of the anisotropic manybody system. 
The Hamiltonian describing the relative motion of two polarized dipoles of mass $m$ moving on the plane reads as

$$
\hat{H}_{2}=-\frac{\hbar^{2}}{2 M} \nabla^{2}+\frac{C_{d d}}{4 \pi}\left[\frac{1-3 \lambda^{2} \cos ^{2} \theta}{r^{3}}\right],
$$

where $M=m / 2$ is the reduced mass. In the following and throughout all this paper, we use dimensionless variables scaled according to the characteristic dipolar length $r_{d}=$ $m C_{d d} / 4 \pi \hbar^{2}$ and energy $\epsilon_{d}=\hbar^{2} / m r_{d}^{2}$.

The two linearly independent solutions of the zero-energy Schrödinger equation (SE) for the isotropic case are the building blocks we need in order to generate the Green's function that we will use afterward to solve the anisotropic case. Setting $\lambda=0$, the SE of the relative motion of the two dipoles at zero energy reduces to

$$
-\nabla^{2} \varphi+\frac{1}{r^{3}} \varphi=0
$$

and the general solution of this equation can be expanded in partial waves as

$$
\varphi(r, \theta)=\sum_{n=0}^{\infty} \varphi_{n}(r) \cos (n \theta),
$$

where the wave function of each separate mode $\varphi_{n}(r)$ satisfies

$$
-\frac{1}{r} \frac{d}{d r}\left(r \frac{d \varphi_{n}}{d r}\right)+\left(\frac{n^{2}}{r^{2}}+\frac{1}{r^{3}}\right) \varphi_{n}=0,
$$

which is a modified Bessel equation for $\varphi_{n}(2 / \sqrt{r})$. The two linearly independent solutions of Eq. (7) are $K_{2 n}(2 / \sqrt{r})$ and $I_{2 n}(2 / \sqrt{r})$ [31], and these two functions enter in the Green's function that we write below. The zero-energy solution of the SE requires the condition $\varphi_{2 n}(r=0)$ to be zero, and that discards the $I_{2 n}(2 / \sqrt{r})$ contributions. Apart from a normalization constant, the zero-energy solution of the $\lambda=0$ problem becomes then

$$
\varphi_{n}(r)=K_{2 n}\left(\frac{2}{\sqrt{r}}\right) .
$$

The physical solution of the isotropic case corresponds to $n=$ 0 as otherwise anisotropic contributions would dominate at large distances since $K_{2 n}(2 / \sqrt{r})$ grows as $r^{n}$ when $r \rightarrow \infty$. In $1 \mathrm{D}$ and $3 \mathrm{D}$, the knowledge of the $E=0$ solution allows one to immediately obtain an exact expression for the $s$-wave scattering length $a_{s}$. The description of two-body scattering in $2 \mathrm{D}$ is more involved since the low-energy expansion of the scattering amplitude diverges at low energies, thus introducing additional problems not found in higher and lower dimensions. The scattering length $a_{s}$ can, however, be defined to be equal to the position of the node of the asymptotic form of the zero-energy two-body wave function. This definition has the additional advantage that it can be used in any dimensions and will therefore be adopted throughout this work $[25,32]$. The large- $r$ behavior of $K_{0}(2 / \sqrt{r})$ is $-\gamma+\frac{1}{2} \ln (r)$ and yields the well-known expression

$$
a_{s}=e^{2 \gamma} \approx 3.17222 \ldots,
$$

where $\gamma$ is Euler's gamma constant.
The SE describing the anisotropic case can be cast in the form

$$
-\nabla^{2} \phi+\frac{1}{r^{3}} \phi=\frac{3 \lambda^{2} \cos ^{2} \theta}{r^{3}} \phi,
$$

and the general solution for $\lambda \neq 0$ can be derived from the Green's function corresponding to $\lambda=0$, which fulfills the equation

$$
\left(-\nabla^{2}+\frac{1}{r^{3}}\right) G\left(\mathbf{r}, \mathbf{r}^{\prime}\right)=\delta\left(\mathbf{r}-\mathbf{r}^{\prime}\right),
$$

leading to

$$
\phi(\mathbf{r})=\varphi_{0}(r)+3 \lambda^{2} \int d \mathbf{y} \frac{\cos ^{2} \theta_{y}}{y^{3}} G(\mathbf{r}, \mathbf{y}) \phi(\mathbf{y})
$$

with $\varphi_{0}(r)=K_{0}(2 / \sqrt{r})$ the $\lambda=0$ solution as described above.

Equation (11) can be solved by expanding the Green's function in partial waves as before:

$$
G\left(\mathbf{r}, \mathbf{r}^{\prime}\right)=\frac{1}{2 \pi} g_{0}\left(r, r^{\prime}\right)+\frac{1}{\pi} \sum_{n=1}^{\infty} g_{n}\left(r, r^{\prime}\right) \cos \left[n\left(\theta-\theta^{\prime}\right)\right],
$$

where

$$
g_{n}\left(r, r^{\prime}\right)= \begin{cases}2 K_{2 n}\left(\frac{2}{\sqrt{r}}\right) I_{2 n}\left(\frac{2}{\sqrt{r^{\prime}}}\right) & \text { if } r<r^{\prime}, \\ 2 I_{2 n}\left(\frac{2}{\sqrt{r}}\right) K_{2 n}\left(\frac{2}{\sqrt{r^{\prime}}}\right) & \text { if } r>r^{\prime}\end{cases}
$$

satisfies the boundary condition $g_{n}\left(r=0, r^{\prime}\right)=g_{n}\left(r, r^{\prime}=\right.$ $0)=0$ while keeping it bounded at large distances. The general solution of the Fredholm integral equation (12) admits a series expansion in powers of $\lambda^{2}$ :

$$
\phi(\mathbf{r})=\sum_{k=0}^{\infty} \lambda^{2 k} \phi^{(k)}(\mathbf{r}),
$$

where each $\phi^{(k)}(\mathbf{r})$ satisfies the recurrence relation

$$
\phi^{(k+1)}(\mathbf{r})=3 \lambda^{2} \int d \mathbf{y} \frac{\cos ^{2} \theta_{y}}{y^{3}} G(\mathbf{r}, \mathbf{y}) \phi^{(k)}(\mathbf{y}) .
$$

When $\phi^{(k)}(\mathbf{r})$ is further expanded in partial waves and the Bose symmetry is taken into account,

$$
\phi^{(k)}(r, \theta)=\sum_{n=0}^{\infty} \phi_{2 n}^{(k)}(r) \cos (2 n \theta),
$$

the coupling between the different angular momentum channels produced by the $\cos ^{2} \theta$ term of the interaction emerges and the radial functions satisfy the following recurrence relations for even $n$ :

$$
\begin{aligned}
\phi_{n}^{(k+1)}(r)= & \frac{3 \lambda^{2}}{4} \int_{0}^{\infty} d y \frac{g_{n}(r, y)}{y^{2}}\left[\phi_{n+2}^{(k)}(y)\right. \\
& \left.+2 \phi_{n}^{(k)}(y)+\phi_{|n-2|}^{(k)}(y)\right],
\end{aligned}
$$

which can be solved iteratively starting from $\phi_{0}^{(0)}(r)=\varphi_{0}(r)$. From this expression, one sees that by adding successive orders in $\lambda^{2}$ to the series expansion of $\phi(\mathbf{r})$, more angular momentum channels couple together. As in the regime considered, the interaction is fully repulsive $\lambda<1 / \sqrt{3}$ and makes $\lambda^{2}$ a small parameter that we can use in a series expansion of the solution. 


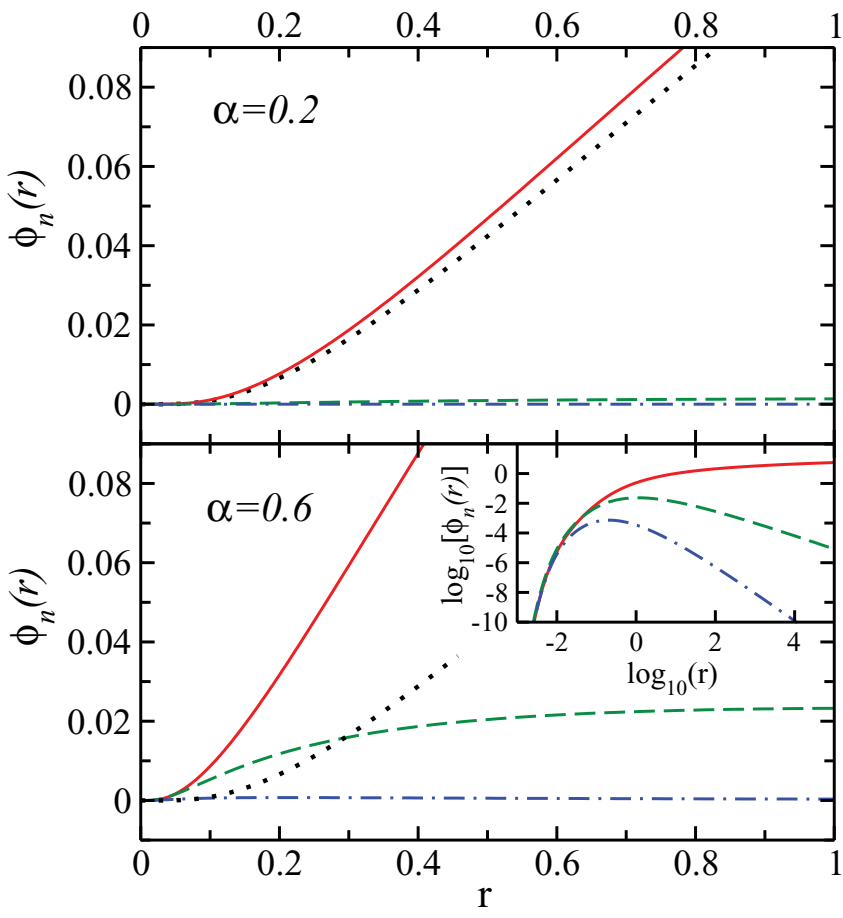

FIG. 2. (Color online) Lowest-order partial wave contributions to the zero-energy two-body scattering wave function. The upper and lower panels show $\phi_{0}^{(0)}=K_{0}(2 / \sqrt{r})$ (black dotted line), $\phi_{0}(r)$ (red solid line), $\phi_{2}(r)$ (green dashed line), and $\phi_{4}(r)$ (blue dotted-dashed line) for the polarization angles $\alpha=0.2$ and 0.6 . The inset show the $\alpha=0.6$ curves in logarithmic scale.

In fact, it can be shown from the previous expressions that $\phi_{2 n}^{(k)}(r)=0$ for $2 n>k$, and that, therefore, the lowest-order contribution to the $n$th mode is $\lambda^{2 n}$. By adding $\phi_{2 n}^{(k)}(r)$ for all $k$ and fixed $n$, one recovers $\phi_{2 n}(r)$, the complete $2 n$th mode contribution to $\phi(\mathbf{r})$. We thus find

$$
\phi_{2 n}(r)=\sum_{k=n}^{\infty} \lambda^{2 k} \phi_{2 n}^{(k)}(r),
$$

which means that, up to a given order $\lambda^{2 k}$, the total wave function $\phi(\mathbf{r})$ has contributions coming only from channels $n=0,2, \ldots, 2 k$.

Figure 2 shows the lowest-order partial wave contributions corresponding to the two polarization angles $\alpha=0.2$ (upper panel) and $\alpha=0.6$ (lower panel), the latter being the largest angle considered in this work. The latter angle is fairly close to the critical angle $\alpha_{c}=0.615$, where the interaction ceases to be purely repulsive. At $\alpha=0.6$, the contribution of the $n \neq 0$ modes is expected to be larger than for any lower angle. This is clearly seen from the figure, where the $\lambda^{2}$ corrections to the $n=0$ and 2 partial waves are shown (red solid and green dashed lines), as well as the leading $\lambda^{4}$ correction corresponding to the $n=0$ mode. It is clear from Eqs. (14)-(18) and the positiveness of the modified Bessel functions that every radial contribution $\phi_{2 n}^{(k)}(r)$ to the two-body wave function is also positive, as seen for the lowest mode contributions in the figure. It is also apparent that the lower the angle, the smaller the correction to the $\alpha=0$ solution $\varphi(r)$ is, as expected. Despite the fact that the series expansion

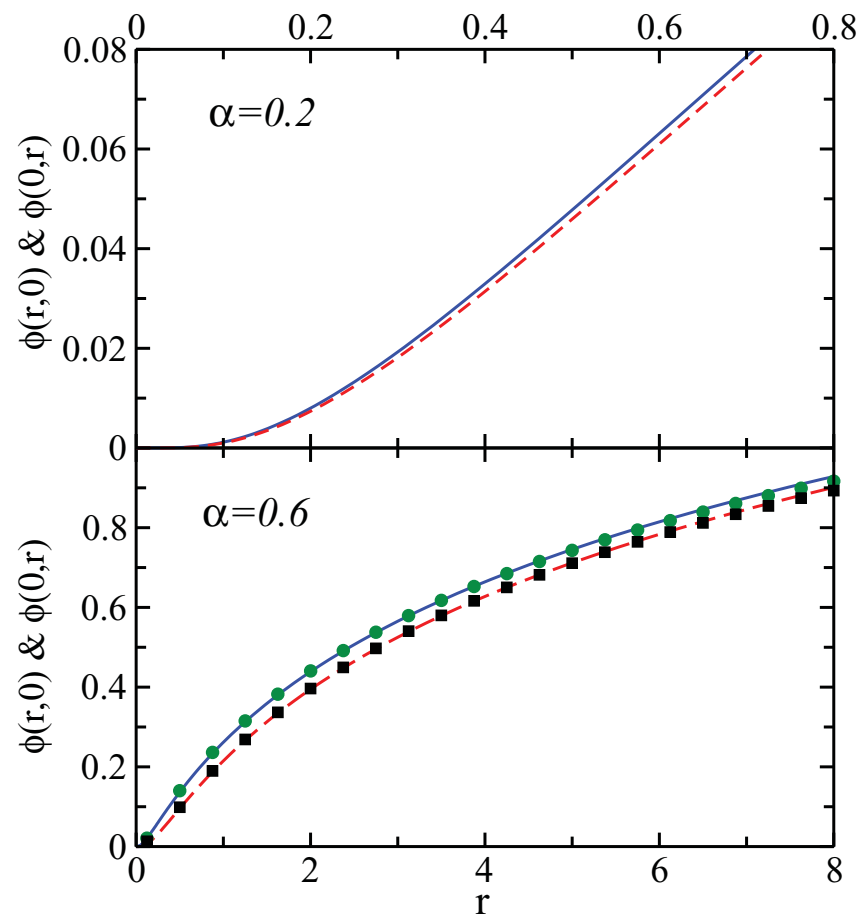

FIG. 3. (Color online) Cuts of the zero-energy two-body scattering wave function describing the relative motion of two dipoles. The blue solid line and the red dashed line correspond to the cuts along the $x$ and $y$ axes, respectively. The green circles and black squares are the prediction of the optimal Jastrow factor obtained from the solution of the HNC/0 Euler equations for a value of the gas parameter $x=0.01$.

of the two-body solution $\phi(\mathbf{r})$ is, in general, alternating due to the cosine terms, the total two-body wave function does not change sign as the interaction is everywhere repulsive, thus making the $E=0$ scattering solution be the ground state.

The effect of the anisotropy on the ground-state wave function is seen in Fig. 3, where two cuts, one along the $x$ axis (contained in the plane formed by the polarization vector and the $z$ axis) and another in the perpendicular direction ( $y$ axis), are depicted for the two polarization angles $\alpha=0.2$ and 0.6. These cuts coincide with the directions where the interaction is least and most repulsive, respectively. As it can be seen, anisotropic effects are visible in both cases, but are more pronounced at high polarization angles. The inset in Fig. 2 shows that the $m=0$ mode dominates at very large distances as expected, making the asymptotic wave function be isotropic. From $\phi_{0}(r)$, one can extract the scattering length $a_{s}(\lambda)$ of the anisotropic dipolar interaction, which is given by the node of its asymptotic $r \rightarrow \infty$ form. An analytic approximation to $a_{s}(\lambda)$ can be easily obtained by recalling that every mode $\phi_{n}(r)$ contributes to order $\lambda^{2 n}$ and that, therefore, the anisotropy enters at order $\lambda^{2}$. Direct inspection of the modes expansion of the the Schrödinger equation for $\phi(\mathbf{r})$ reveals that $\phi_{0}(r)$ and $\phi_{2}(r)$ are related according to

$$
-\frac{1}{r} \frac{d}{d r}\left(r \frac{d \phi_{0}}{d r}\right)+\frac{1}{r^{3}} \phi_{0}=\frac{3 \lambda^{2}}{2 r^{3}}\left[\phi_{0}(r)+\frac{1}{2} \phi_{2}(r)\right] .
$$


An approximation of order $\lambda^{2}$ to $a_{s}(\lambda)$ can be obtained by keeping only the $\phi_{0}(r)$ mode on the right-hand side of this equation:

$$
-\frac{1}{r} \frac{d}{d r}\left(r \frac{d \phi_{0}^{(0)}}{d r}\right)+\frac{1}{r^{3}} \phi_{0}^{(0)}=\frac{3 \lambda^{2}}{2 r^{3}} \phi_{0}^{(0)}(r),
$$

which once again is a modified Bessel equation with the general solution

$$
\begin{aligned}
\phi_{0}^{(0)}(r)= & \mathcal{N}\left[K_{0}\left(2 \sqrt{\frac{1-\frac{3 \lambda^{2}}{2}}{r}}\right)\right. \\
& \left.+B(\lambda) I_{0}\left(2 \sqrt{\frac{1-\frac{3 \lambda^{2}}{2}}{r}}\right)\right]
\end{aligned}
$$

with $\mathcal{N}$ a normalization constant. In this expression, $B(\lambda)$ is an unknown function of $\lambda^{2}$ according to the parity of the Hamiltonian under the $\lambda \rightarrow-\lambda$ transformation. Furthermore, $B(0)=0$ so that one recovers the isotropic solution given in Eq. (9). Hence, $B(\lambda)=b_{2} \lambda^{2}$ to order $\lambda^{2}$, with $b_{2}$ a constant. In the asymptotic $r \rightarrow \infty$ regime, $I_{0} \approx 1$ and one can compare the expansion to order $\lambda^{2}$ of the above expression to the expression of $\phi_{0}(r)$ to the same order obtained from the integration of the Green's function done before. This yields $b_{2}=0$ and one has

$\left.\phi_{0}(r \rightarrow \infty) \rightarrow K_{0}\left(2 \sqrt{\frac{1-\frac{3 \lambda^{2}}{2}}{r}}\right)\right|_{r \rightarrow \infty} \approx \frac{1}{2} \ln \frac{r}{a_{s}(\lambda)}$

with $a_{s}(\lambda)$ the $s$-wave scattering length

$$
a_{s}(\lambda)=e^{2 \gamma}\left(1-\frac{3 \lambda^{2}}{2}\right)
$$

This expression is accurate up to order $\lambda^{2}$, so one could expect it to provide a reliable prediction only at small polarization angles. This turns out not to be the case, and in Fig. 4, we show the comparison of this approximation to the exact result

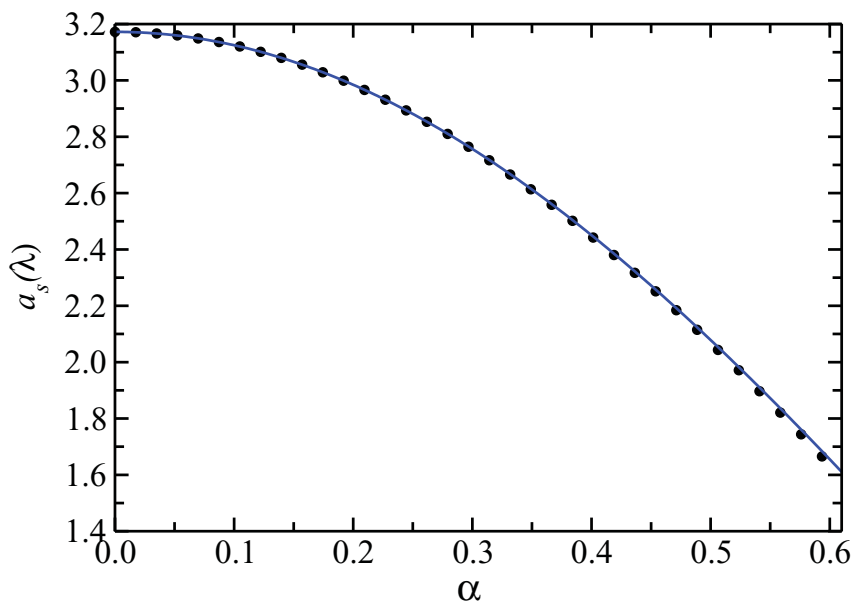

FIG. 4. (Color online) $s$-wave scattering length $a_{s}[\lambda=\sin (\alpha)]$ as a function of the polarization angle $\alpha$. The blue solid line and the black dots correspond to the exact numerical solution and the $\lambda^{2}$ approximation of Eq. (23), respectively. obtained by numerically finding the node of the asymptotic $m=0$ wave function, which is isotropic and dominates the large distance behavior of $\phi(\mathbf{r})$. As can be seen from the figure, the approximation works surprisingly well up to the critical angle $\alpha_{c}$, where the interaction ceases to be fully repulsive. Deviations increase with increasing polarization angle, but even at $\alpha=\alpha_{c}$, the separation between the approximation in Eq. (23) and the exact numerical estimation is less than $3 \%$.

\section{MANY-BODY DESCRIPTION}

In this section, we extend the previous discussion and analyze, using DMC and VMC methods, the most relevant ground-state properties of a homogeneous and anisotropic gas of bosonic dipoles with a polarization angle $\alpha$ lower than the critical angle $\alpha_{c}=0.615$. We stick to the low-density limit where the system, characterized by a fully repulsive and anisotropic interaction, remains in a stable, gaseous phase. One of the most relevant quantities to analyze at low densities is the total energy per particle of the gas and its universal scaling properties. Quite a lot of work has been devoted in the past to that question, including both 3D [20,33-35] and 2D [24-30] systems. However, little has been discussed about the same properties in anisotropic systems as the dipolar gas considered here. We analyze the impact of the polarization angle $\alpha$ formed by the dipoles on the universality scaling law exhibited by other isotropic, short-ranged interactions.

The Hamiltonian of the system of fully polarized dipoles, written in the same dipolar units used in the preceding section, becomes

$$
H=-\frac{1}{2} \sum_{j=1}^{N} \nabla_{j}^{2}+\sum_{i<j} \frac{1-3 \lambda^{2} \cos ^{2} \theta_{i j}}{r_{i j}^{3}},
$$

with $\lambda=\sin \alpha$, and $r_{i j}$ and $\theta_{i j}$ the distance and angle formed by dipoles $i$ and $j$, respectively, measured on the plane.

The leading ground-state quantities describing the lowdensity static properties of the system can be obtained using different techniques. In this work, we stick to diffusion and variational Monte Carlo methods, widely used nowadays in the analysis of weakly and strongly correlated systems. Variational Monte Carlo samples stochastically a trial wave function and yields an upper bound to the real ground-state energy of the system. On the other hand, diffusion Monte Carlo uses also a trial wave function to guide the sampling process, but removes the contributions from excited states to finally yield statistically exact energies. In both cases, however, a suitable variational wave function is required. The quality of the results is directly related to the quality of the wave function employed in the VMC case, while DMC is far less demanding and any reasonable guiding function can be used as long as it is not orthogonal to the true ground state. But, even in DMC, a high-quality wave function makes the method converge faster and with smaller variance toward the exact result. Consequently, seeking for a good trial many-body wave function $\Psi\left(\mathbf{r}_{1}, \mathbf{r}_{2}, \ldots, \mathbf{r}_{N}\right)$ is always desirable.

In this paper, we use a model wave function of the Jastrow form

$$
\Psi\left(\mathbf{r}_{1}, \mathbf{r}_{2}, \ldots, \mathbf{r}_{N}\right)=\prod_{i<j} f\left(\mathbf{r}_{i j}\right)
$$


TABLE I. DMC and VMC energies per particle as a function of the gas parameter $x=n a^{2}$.

\begin{tabular}{|c|c|c|c|c|c|c|}
\hline \multirow[b]{2}{*}{$x$} & \multicolumn{2}{|c|}{$\alpha=0.2$} & \multicolumn{2}{|c|}{$\alpha=0.4$} & \multicolumn{2}{|c|}{$\alpha=0.6$} \\
\hline & $\mathrm{DMC}$ & VMC & DMC & VMC & DMC & VMC \\
\hline $10^{-7}$ & $4.271(61) \times 10^{-9}$ & $4.268(92) \times 10^{-9}$ & $6.469(62) \times 10^{-9}$ & $6.490(24) \times 10^{-9}$ & $1.414(62) \times 10^{-8}$ & $1.429(75) \times 10^{-8}$ \\
\hline $5 \times 10^{-7}$ & $2.386(24) \times 10^{-8}$ & $2.389(90) \times 10^{-8}$ & $3.602(70) \times 10^{-8}$ & $3.633(91) \times 10^{-8}$ & $7.888(15) \times 10^{-8}$ & $7.931(45) \times 10^{-8}$ \\
\hline $10^{-6}$ & $5.030(32) \times 10^{-8}$ & $5.044(91) \times 10^{-8}$ & $7.614(21) \times 10^{-8}$ & $7.631(36) \times 10^{-8}$ & $1.664(50) \times 10^{-7}$ & $1.690(86) \times 10^{-7}$ \\
\hline $5 \times 10^{-6}$ & $2.868(24) \times 10^{-7}$ & $2.874(23) \times 10^{-7}$ & $4.317(70) \times 10^{-7}$ & $4.360(89) \times 10^{-7}$ & $9.448(93) \times 10^{-7}$ & $9.472(85) \times 10^{-7}$ \\
\hline $10^{-5}$ & $6.105(64) \times 10^{-7}$ & $6.135(87) \times 10^{-7}$ & $9.271(41) \times 10^{-7}$ & $9.312(22) \times 10^{-7}$ & $2.032(90) \times 10^{-6}$ & $2.011(92) \times 10^{-6}$ \\
\hline $5 \times 10^{-5}$ & $3.584(31) \times 10^{-6}$ & $3.596(27) \times 10^{-6}$ & $5.405(15) \times 10^{-6}$ & $5.450(94) \times 10^{-6}$ & $1.180(40) \times 10^{-5}$ & $1.199(81) \times 10^{-5}$ \\
\hline $10^{-4}$ & $7.744(61) \times 10^{-6}$ & $7.768(72) \times 10^{-6}$ & $1.170(41) \times 10^{-5}$ & $1.177(30) \times 10^{-5}$ & $2.542(88) \times 10^{-5}$ & $2.579(84) \times 10^{-5}$ \\
\hline $5 \times 10^{-4}$ & $4.734(49) \times 10^{-5}$ & $4.757(48) \times 10^{-5}$ & $7.124(93) \times 10^{-5}$ & $7.205(59) \times 10^{-5}$ & $1.555(62) \times 10^{-4}$ & $1.567(73) \times 10^{-4}$ \\
\hline $10^{-3}$ & $1.046(16) \times 10^{-4}$ & $1.051(31) \times 10^{-4}$ & $1.577(33) \times 10^{-4}$ & $1.590(58) \times 10^{-4}$ & $3.425(30) \times 10^{-4}$ & $3.467(23) \times 10^{-4}$ \\
\hline $5 \times 10^{-3}$ & $6.776(61) \times 10^{-4}$ & $6.807(74) \times 10^{-4}$ & $1.018(90) \times 10^{-3}$ & $1.029(58) \times 10^{-3}$ & $2.222(51) \times 10^{-3}$ & $2.240(26) \times 10^{-3}$ \\
\hline $10^{-2}$ & $1.532(20) \times 10^{-3}$ & $1.551(31) \times 10^{-3}$ & $2.316(31) \times 10^{-3}$ & $2.337(23) \times 10^{-3}$ & $5.036(55) \times 10^{-3}$ & $5.067(97) \times 10^{-3}$ \\
\hline $5 \times 10^{-2}$ & $1.077(11) \times 10^{-2}$ & $1.085(29) \times 10^{-2}$ & $1.616(9) \times 10^{-2}$ & $1.634(18) \times 10^{-2}$ & $3.517(74) \times 10^{-2}$ & $3.544(62) \times 10^{-2}$ \\
\hline $10^{-1}$ & $2.534(29) \times 10^{-2}$ & $2.572(67) \times 10^{-2}$ & $3.774(42) \times 10^{-1}$ & $3.840(66) \times 10^{-2}$ & $8.235(21) \times 10^{-2}$ & $8.292(21) \times 10^{-2}$ \\
\hline $5 \times 10^{-1}$ & $1.947(14) \times 10^{-1}$ & $1.962(54) \times 10^{-1}$ & $2.908(28) \times 10^{-1}$ & $2.938(41) \times 10^{-1}$ & $6.311(33) \times 10^{-1}$ & $6.347(32) \times 10^{-1}$ \\
\hline
\end{tabular}

where the two-body correlation factor $f\left(\mathbf{r}_{i j}\right)=f\left(\mathbf{r}_{i}-\mathbf{r}_{j}\right)$ depends on the position vector linking particles $i$ and $j$. One significant difference between this Jastrow factor and the ones most commonly employed in the analysis of other condensed matter systems is that, due to the anisotropic character of the interaction, $f(\mathbf{r})$ depends explicitly on the whole $\mathbf{r}$ vector and not only on its magnitude. In this way, the wave function in Eq. (25) describes a homogeneous but anisotropic system as the one under study.

At low densities, the zero-energy scattering solution of the two-body problem greatly influences the structural properties of the gas. For that reason, we use as a Jastrow factor the anisotropic solution of the relative motion of two dipoles on the plane derived in the preceding section. The $n=0$ mode of this wave function is matched at some healing distance $\xi$ with the symmetrized form of a phononic wave function $f_{\xi}(r)=\exp (-C / r)$ [36], taking both $\xi$ and $C$ as variational parameters and imposing the continuity of $f(r)$ and $f^{\prime}(r)$ at $r=\xi$. The $n>0$ modes of the two-body problem, inducing the anisotropy of $\Psi\left(\mathbf{r}_{1}, \mathbf{r}_{2}, \ldots, \mathbf{r}_{N}\right)$ in Eq. (25), decay to zero at large distances and so their influence at the boundaries of the simulation box is marginally small. Alternatively, the optimal Jastrow factor corresponding to the many-body problem can be obtained from the solution of the HNC/0 Euler-Lagrange equations [37]. Although not exact, the optimized HNC/0 solution gives an accurate variational description of quantum Bose systems and captures most of the short- and long-range features of the exact ground-state wave function. For the sake of comparison, we also show in Fig. 3 the optimized HNC/0 Jastrow factor (black and green symbols) at $x=0.01$ and polarization angle $\alpha=0.6$. The comparison indicates that the two-body solution provides an accurate description of the two-body correlation factor, which becomes even better as the gas parameter is reduced. We have checked that the HNC/0 Jastrow factor and the solution of the two-body problem are in very good agreement in the whole range of gas parameter values considered in this work.

Table I lists both the VMC and DMC energies obtained from the Jastrow trial wave function of Eq. (25) for the polarization angles $\alpha=0.2,0.4$, and 0.6 . Notice that the energies in the table are given for fixed $x$ and different polarization angles, and since the scattering length varies with $\alpha$, the densities change accordingly. A direct measure of the quality of the variational model is given by the separation between these two measures (VMC and DMC), and one can check that the relative difference in energies is always of the order of $1 \%$ or $2 \%$. Other than that, the energy is an increasing function of the gas parameter that yields appreciably different results for different polarization angles. These energies can be used to check the influence of the anisotropic character of the dipolar interaction on the universality scaling property fulfilled by the energy per particle of homogeneous and isotropic systems in 2D. In order to do that, one has to express the total energy per particle in units of $\hbar^{2} / 2 m a^{2}$ with $a$ the scattering length. This is achieved by multiplying the energies in Table I (expressed in dipolar units) by $2 a_{s}^{2}(\lambda)$, with $a_{s}(\lambda)$ the scattering length for the corresponding polarization angle. Figure 5 shows the ratio of the energy per particle in units of $\hbar^{2} / 2 m a^{2}$ to the mean-field prediction of Eq. (3) for the three polarization angles $\alpha=$ $0.0,0.4$, and 0.6. As it can be seen, expressed in scattering length units, all curves corresponding to different polarization angles merge into a single curve, with very small deviations that are not easily resolved even at the highest values of gas parameters $x$ considered in this work. That means that the anisotropy of the interaction, present in the wave function, does not appreciably affect the energy per particle in the low-density regime analyzed in this work. We conclude that the difference in energy values shown in Table I for fixed $x$ and varying polarization angles are to be mostly attributed to the different density $n=x / a^{2}$ in each case.

Figure 5 also shows the universal curve including beyond mean-field effects of Ref. [29] and the optimized HNC/0 prediction for a gas of hard disks of Ref. [30]. As it can be seen, the universal and the hard disk curves are close to each other while the dipole curves remain closer to the mean-field prediction $\epsilon_{m f}(x)$ as the gas parameter is raised. Starting at $x \sim 0.05$, the dipole curves bend downward and the energy deviates significantly from $\epsilon_{m f}(x)$. In any case, it is clear from 


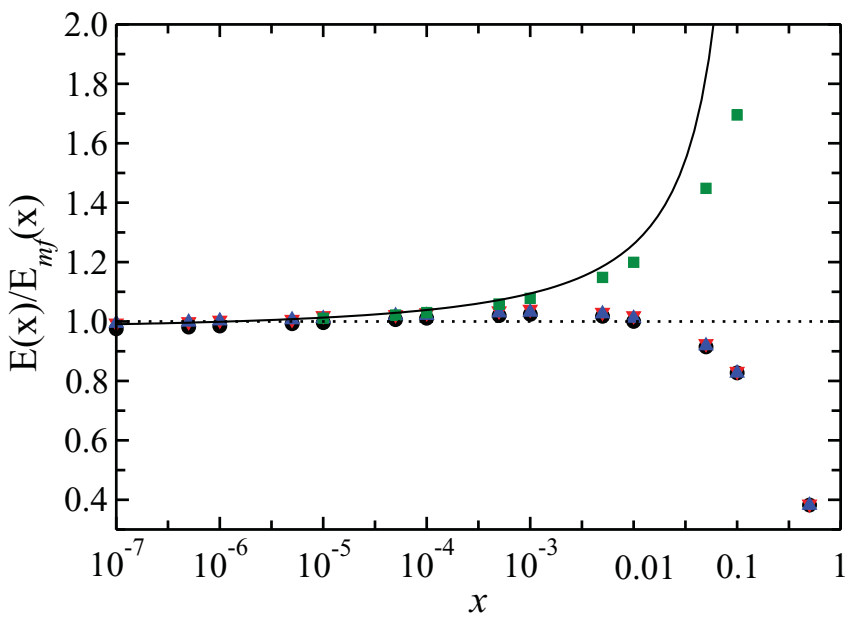

FIG. 5. (Color online) Ratio of the energy per particle of the gas of dipoles for different polarization angles to the mean-field prediction of Eq. (3). Black circles, red triangles, and blue triangles correspond to $\alpha=0.2,0.4$, and 0.6 , respectively. The green squares are the optimized HNC/EL energies for hard disks of Ref. [35], while the solid line is the universal curve of Ref. [32]. The dotted line corresponds to the mean-field prediction.

the figure that the universality regime where the energy per particle depends only on the gas parameter of the interaction is left much before anisotropic effects have an appreciable impact on the energy of the dipolar gas.

The anisotropic character of the dipolar interaction has a direct influence on the ground-state wave function that is reflected in the ground-state expectation value of any many-

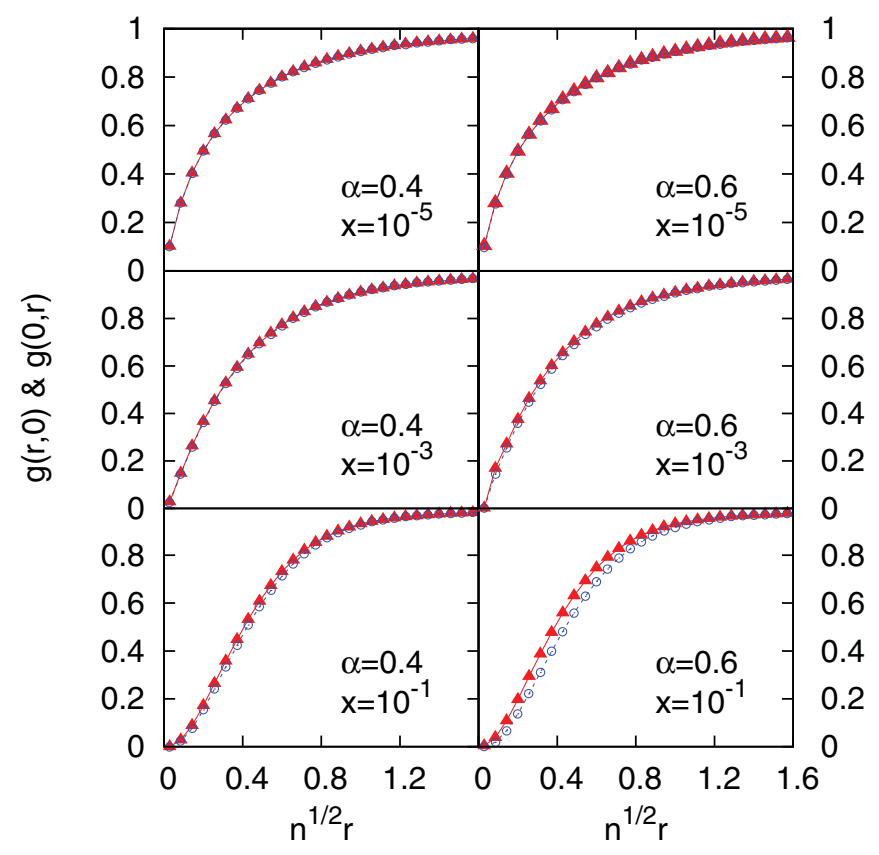

FIG. 6. (Color online) Pair distribution function for $\alpha=0.4$ and $\alpha=0.6$ and three values of the gas parameter. The red and blue curves (triangles and open circles, respectively) show the two cuts $g(r, 0)$ and $g(0, r)$, respectively.

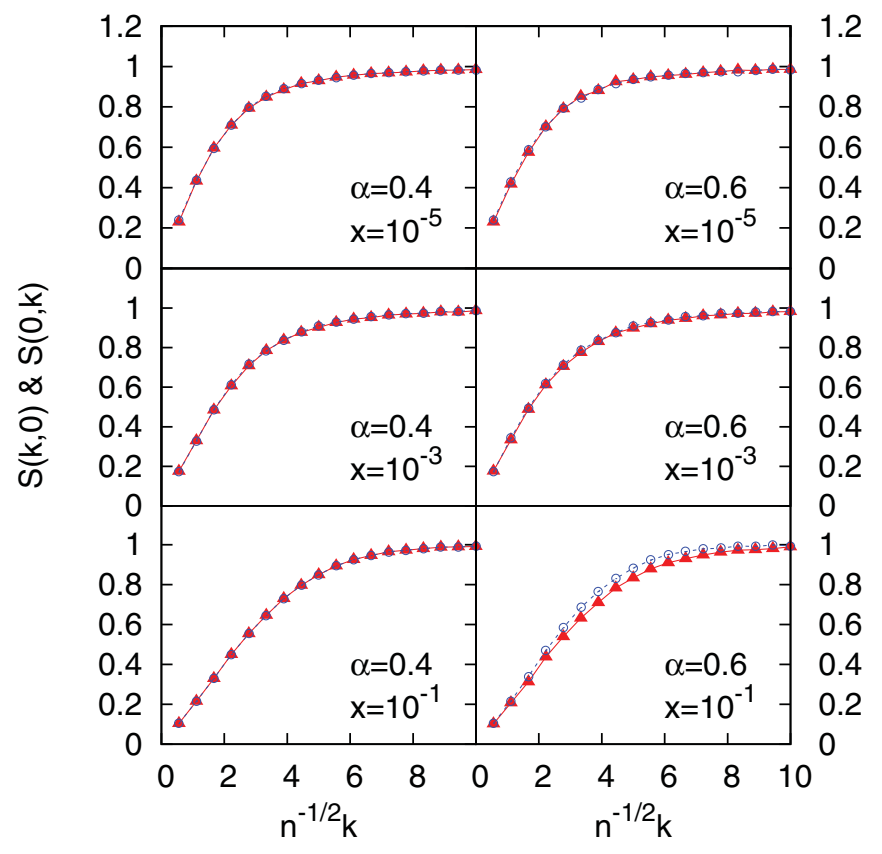

FIG. 7. (Color online) Static structure function for polarization angles $\alpha=0.4$ and $\alpha=0.6$ for $x=10^{-5}, 10^{-3}$ and $10^{-1}$. The red and blue curves (triangles and open circles, respectively) show the two cuts $S(k, 0)$ and $S(0, k)$, respectively.

body operator. Figures 6 and 7 show pure DMC estimations [38] of the pair distribution function $g(\mathbf{r})$ and its Fourier transform, the static structure factor $S(\mathbf{k})$, for two values of the polarization angle $\alpha=0.4$ and 0.6 (left and right panels), and three values of the gas parameter $x=10^{-5}, 10^{-3}$, and $10^{-1}$ (top to bottom). Notice that in both figures the horizontal axis has been scaled with the square root of the density for a better comparison. Due to the symmetries of the Hamiltonian, the complete $g(\mathbf{r})$ and $S(\mathbf{k})$ functions vary continuously on the plane, but the pattern on the first quadrant is repeated and reflected on the other three. The figures show only the two cuts along the perpendicular and parallel directions with respect to the polarization plane, corresponding to the lines where the interaction is most and least repulsive, respectively. As it can be seen, and in agreement with what one would expect, the effect of the anisotropy is more clearly seen at higher polarization angles and for large values of the gas parameter, being maximal for $\alpha=0.6$ and $x=10^{-1}$. For fixed $\alpha$, the separation between $g(r, 0)$ and $g(0, r)$ is enhanced with increasing $x$, as happens with $S(k, 0)$ and $S(0, k)$. Accordingly, and for a given $x$, the separation between the curves also increases when the polarization angle is raised. In any case, it is remarkable how the anisotropy present in $g(\mathbf{r})$ and $S(\mathbf{k})$ changes with the polarization angle as can be seen from the figures at large $x$, while the total energies per particle are almost the same when properly scaled with the scattering length. This points toward a delicate balance between the kinetic and potential contributions, which change with $\alpha$ but keep their sum constant once expressed in scattering length units.

The last quantity analyzed in this paper is the one-body density matrix $\rho_{1}\left(\mathbf{r}_{1}, \mathbf{r}_{1}^{\prime}\right)$, which provides a measure of the 
overlap between two instances of the ground-state wave function when one particle is shifted from its initial position at $\mathbf{r}_{1}$ to a new position at $\mathbf{r}_{1}^{\prime}$ :

$$
\begin{aligned}
& \rho_{1}\left(\mathbf{r}_{1}, \mathbf{r}_{1}^{\prime}\right) \\
& \quad=N \frac{\int d \mathbf{r}_{2} \ldots \mathbf{r}_{N} \Psi_{0}\left(\mathbf{r}_{1}, \mathbf{r}_{2}, \ldots, \mathbf{r}_{N}\right) \Psi_{0}\left(\mathbf{r}_{1}^{\prime}, \mathbf{r}_{2}, \ldots, \mathbf{r}_{N}\right)}{\int d \mathbf{r}_{1} d \mathbf{r}_{2} \ldots \mathbf{r}_{N} \Psi_{0}^{2}\left(\mathbf{r}_{1}, \mathbf{r}_{2}, \ldots, \mathbf{r}_{N}\right)} .
\end{aligned}
$$

In the case of translationally invariant systems as the one under study, the one-body density matrix depends on its arguments only through their difference and thus $\rho_{1}\left(\mathbf{r}_{1}, \mathbf{r}_{1}^{\prime}\right)=\rho_{1}\left(\mathbf{r}_{1}-\right.$ $\left.\mathbf{r}_{1}^{\prime}, 0\right) \equiv \rho_{1}\left(\mathbf{r}_{11^{\prime}}\right)$ Additionally, if the interaction is isotropic, $\rho_{1}$ depends only on the magnitude of its argument $r_{11^{\prime}}=\left|\mathbf{r}_{11^{\prime}}\right|$ and its large- $r_{11^{\prime}}$ limit measures directly the condensate fraction $n_{0}$, which is proportional to the number of particles in the BoseEinstein condensate. In the present case, however, the system is homogeneous but not isotropic so $\rho_{1}\left(\mathbf{r}_{11^{\prime}}\right)$ will depend on the direction of $\mathbf{r}_{11^{\prime}}$. Due to translational invariance, though, momentum is still a good quantum number and one expects condensation to appear at the zero momentum state. In that sense, one can still write the relation between $\rho_{1}\left(\mathbf{r}_{11^{\prime}}\right)$ and the momentum distribution in the form

$$
\rho_{1}\left(\mathbf{r}_{11^{\prime}}\right)=\rho n_{0}+\frac{1}{(2 \pi)^{2}} \int d \mathbf{k} e^{i \mathbf{k} \cdot \mathbf{r}_{11^{\prime}}} \tilde{n}(\mathbf{k}),
$$

where $\tilde{n}(\mathbf{k})$ is the momentum distribution of the noncondensate atoms. The one-body density matrix of the anisotropic gas of Bose dipoles can be further expanded in partial waves

$$
\rho_{1}(\mathbf{r})=\sum_{m=0}^{\infty} \rho_{1 m}(r) \cos (2 m \theta)
$$

with $\rho_{1 m}(r)$ the radial function corresponding to the $m$ th mode contribution. Notice that, as before, the Bose symmetry restricts the previous sum to even modes only.

Once enough modes $\rho_{1 m}(r)$ are known, one can reconstruct the complete one-body density matrix for all points in the plane. In particular, the cuts along the two directions parallel and perpendicular to the polarization plane, corresponding to $\theta=0$ and $\pi / 2$ in Eq. (28), turn out to be particularly easy to evaluate

$$
\rho_{1}(r, 0)=\sum_{m=0}^{\infty} \rho_{1 m}(r), \quad \rho_{1}(0, r)=\sum_{m=0}^{\infty}(-1)^{m} \rho_{1 m}(r)
$$

and display the maximum difference two cuts along different directions can take at the low densities considered in this work. Figure 8 shows the parallel and perpendicular cuts of $\rho_{1}(\mathbf{r})$ for the polarization angles $\alpha=0.4$ and 0.6 (left and right panels). The upper and lower curves correspond to the gas parameter values $x=10^{-3}$ and $10^{-1}$, respectively. As before, the coordinates on the horizontal axis have been scaled with the density. Similarly to what happens to the other quantities analyzed, only at the highest gas parameter values the effects of the anisotropy start to be visible. This stresses once again the minor role played by the anisotropy at low densities, even in a nondiagonal quantity such as $\rho_{1}\left(\mathbf{r}_{11^{\prime}}\right)$.

The most significant differences in the one-body density matrix for different values of the gas parameter appear at large distances, where $\rho_{1}\left(\mathbf{r}_{11^{\prime}}\right)$ reaches an asymptotic value that can be identified with the condensate fraction $n_{0}$ in isotropic

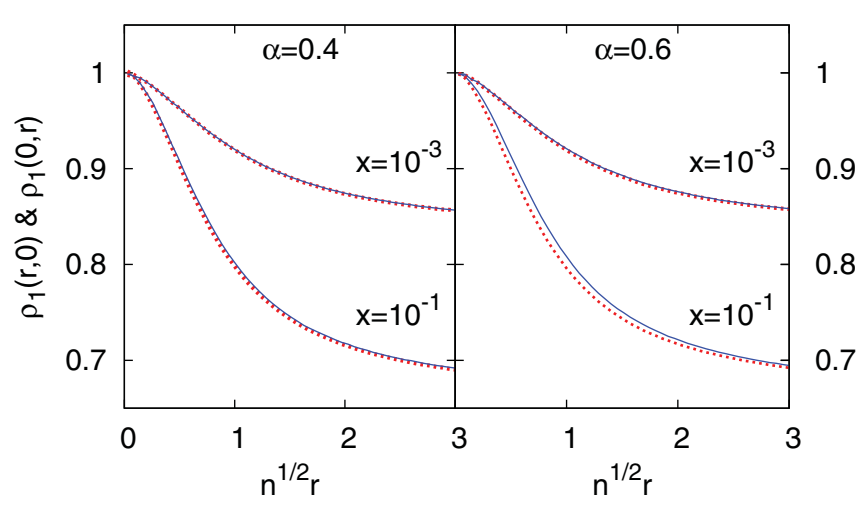

FIG. 8. (Color online) Cuts of the one-body density matrix along the $x$ (red dotted lines) and $y$ (blue solid lines) axes, for the gas parameter values $x=10^{-3}$ and $10^{-1}$ (top to bottom). The curves on the left and right panels correspond to $\alpha=0.4$ and 0.6 , respectively.

systems. When the anisotropic character of the interaction is taken into account, the presence of higher-order partial waves in Eqs. (28) and (29) could, in principle, change this behavior, making the limiting value of $\rho_{1}\left(\mathbf{r}_{11^{\prime}}\right)$ depend on the direction. The role of the different partial waves in that limit can be determined by looking at the momentum distribution of the system, which can be obtained from $\rho_{1}\left(\mathbf{r}_{11^{\prime}}\right)$ by looking at the inverse of Eq. (27):

$$
\begin{aligned}
\tilde{n}(\mathbf{k})= & \int_{0}^{\infty} d r r \int_{0}^{2 \pi} d \theta e^{i k r \cos (\theta-\varphi)}\left[\left(\rho_{10}(z)-\rho n_{0}\right)\right. \\
& \left.+\sum_{m=1}^{\infty} \rho_{1 m}(r) \cos (2 m \theta)\right],
\end{aligned}
$$

with $\varphi$ the angle formed by $\mathbf{k}$ and the $x$ axis. By changing variables $\alpha=\theta-\varphi$, using the Jacobi-Anger expansion of a plane wave in Bessel functions

$$
e^{i k z \cos \alpha}=J_{0}(k z)+2 \sum_{m=1}^{\infty} i^{m} J_{m}(k z) \cos (m \alpha),
$$

and taking into account the orthogonality of the cosine functions in the range $[0,2 \pi]$, one finally finds

$$
\begin{aligned}
\tilde{n}(\mathbf{k})= & \pi \int_{0}^{\infty} J_{0}(k r)\left[\rho_{10}(r)-n_{0}\right] r d r+2 \pi \sum_{m=1}^{\infty}(-1)^{m} \\
& \times \cos (2 m \varphi) \int_{0}^{\infty} J_{2 m}(k r) \rho_{1 m}(r) r d r
\end{aligned}
$$

where the first term on the right is isotropic and constitutes the $m=0$ mode of $\tilde{n}(\mathbf{k})$, while the other terms stand for the $m>0$ contributions. Notice once again that only even modes appear in this expansion.

Requiring $\tilde{n}(\mathbf{k})$ to be finite for all values of $\mathbf{k}$ implies all integrals appearing in Eq. (32) to be finite, a constraint that can only be fulfilled when the functions multiplying the Bessel functions decay to zero at large distances. This condition particularly means that $n_{0}$ can be obtained as the large- $r$ limit of the $m=0$ mode of the one-body density matrix, which is the isotropic contribution to $\rho_{1}\left(\mathbf{r}_{11^{\prime}}\right)$. This is the direct generalization of the usual procedure employed to determine $n_{0}$ in homogeneous and isotropic systems. 


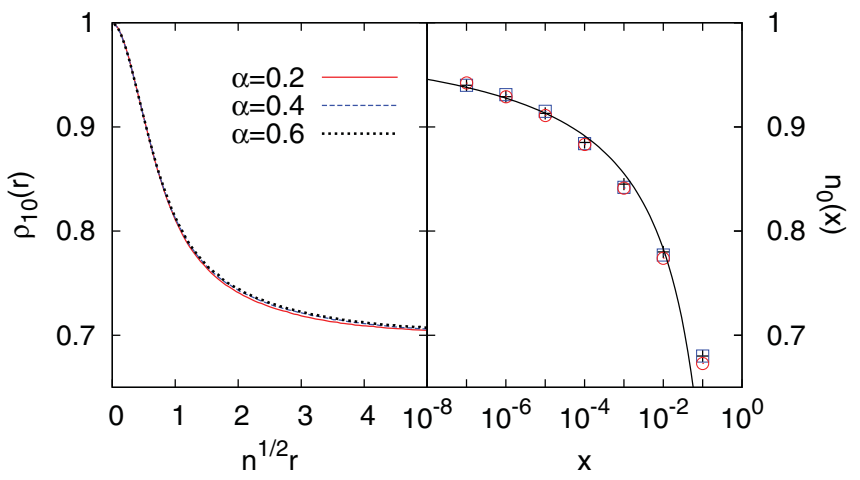

FIG. 9. (Color online) Left panel: isotropic $(m=0)$ contribution to the one-body density matrix at $x=0.1$ for the three polarization angles $\alpha=0.2,0.4$, and 0.6 (red solid, blue dashed, and black dotted lines, respectively). Right panel: condensate fraction $n_{0}$ as a function of the gas parameter for $\alpha=0.2,0.4$, and 0.6 , compared with the Bogoliubov prediction (black line). The color coding for the symbols is the same as in the left panel.

Figure 9 shows on the left panel the $m=0$ mode contribution $\rho_{10}(r)$ for the three polarization angles $\alpha=0.2,0.4$, and 0.6 in terms of the scaled distances $n^{1 / 2} r$ for $x=0.1$. As can be seen from the figure, all three curves are hardly distinguishable, stressing once again that, to a large extent, the physics is governed by the scattering length, which makes the density change for different polarization angles when $x$ is fixed. The right panel in the figure shows the condensate fraction as a function of the gas parameter $x=n a^{2}$, obtained from the $r \rightarrow \infty$ limit of a fit to the long-range asymptotic limit of the $m=0$ partial wave contribution of the one-body density matrix. Up to the highest value of $x$ considered, all three cases yield nearly the same prediction within statistical errors, while differences start to be significant only at $x \approx 0.1$. Therefore, the scaling on the gas parameter is preserved, although moving from $\alpha=0.2$ to 0.6 for fixed $x$ implies a change in density by almost a factor of 2 . The figure also shows the Bogoliubov prediction for an isotropic gas of weakly interacting $2 \mathrm{D}$ bosons

$$
n_{0}(x)=1-\frac{1}{|\ln x|},
$$

which agrees reasonably well with the Monte Carlo prediction up to $x \approx 0.01$, where particle correlations seem to deplete the condensate less effectively than the mean-field model.

\section{SUMMARY AND CONCLUSIONS}

To summarize, in this paper, we have described the groundstate properties of a gas of fully polarized Bose dipoles moving on the $X Y$ plane, where the polarization field forms an angle $\alpha$ with the normal direction. The projection of the polarization vector on the $X Y$ plane defines the $x$ axis, where the potential is softer than in any other direction. In this context, the dipole-dipole interaction defines a critical angle $\alpha_{c} \approx 0.615$ where the potential starts to have attractive contributions. We have solved the zero-energy two-body scattering problem by means of a Green's function and a decomposition of the wave function in partial waves. We have then found the dependence of the $s$-wave scattering length on the polarization angle by inspection of the $m=0$ mode, which dominates at large distances. Equipped with the two-body solution, we have built a variational wave function of the Jastrow type that has been used as a guiding function in a DMC simulation of the gas of polarized dipoles at low densities. We have found that the scaling of the energy in the gas parameter is preserved up to values of $x$ where other isotropic systems deviate significantly. This behavior extends to other relevant ground-state quantities such as the pair distribution function, the static structure factor, and the one-body density matrix, including the condensate fraction, which can be determined from the large-distance asymptotic behavior of its isotropic part.

\section{ACKNOWLEDGMENTS}

This work was partially supported by Grants No. FIS200804403 from DGI (Spain), No. 2009-SGR1003 from the Generalitat de Catalunya (Spain), and No. P23535 form the Austrian Science Fund FWF (Austria).
[1] A. Griesmaier, J. Werner, S. Hensler, J. Stuhler, and T. Pfau, Phys. Rev. Lett. 94, 160401 (2005).

[2] J. Stuhler, A. Griesmaier, T. Koch, M. Fattori, T. Pfau, S. Giovanazzi, P. Pedri, and L. Santos, Phys. Rev. Lett. 95, 150406 (2005).

[3] K.-K. Ni, S. Ospelkaus, M. H. G. de Miranda, A. Pe'er, B. Neyenhuis, J. J. Zirbel, S. Kotochigova, P. S. Julienne, D. S. Jin, and J. Ye, Science 322, 231 (2008).

[4] A. D. Lercher, T. Takekoshi, M. Debatin, B. Schuster, R. Rameshan, F. Ferlaino, R. Grimm, and H.-C. Nägerl, e-print arXiv:1101.1409v1.

[5] S. Ospelkaus, A. Pe'er, K. K. Ni, J. J. Zirbel, B. Neyenhuis, S. Kotochigova, P. S. Julienne, J. Ye, and D. S. Jin, Nat. Phys. 4, 622 (2009).

[6] K.-K. Ni, S. Ospelkaus, D. Wang, G. Quémner, B. Neyenhuis, M. H. G. de Miranda, J. L. Bohn, J. Ye, and D. S. Jin, Nature (London) 464, 1324 (2010).
[7] R. V. Krems, Phys. Chem. Chem. Phys. 10, 4079 (2008).

[8] A. Andre, Nat. Phys. 2, 341 (2006).

[9] D. H. J. O’Dell, S. Giovanazzi, and G. Kurizki, Phys. Rev. Lett. 90, 110402 (2003).

[10] R. M. Wilson, S. Ronen, J. L. Bohn, and H. Pu, Phys. Rev. Lett. 100, 245302 (2008).

[11] L. Santos, G. V. Shlyapnikov, and M. Lewenstein, Phys. Rev. Lett. 90, 250403 (2003).

[12] F. Mazzanti, R. E. Zillich, G. E. Astrakharchik, and J. Boronat, Phys. Rev. Lett. 102, 110405 (2009).

[13] M. Klawunn, A. Pikovski, and L. Santos, Phys. Rev. A 82, 044701 (2010)

[14] C. Ticknor, Phys. Rev. A 80, 052702 (2009).

[15] C. Ticknor, Phys. Rev. A 81, 042708 (2010).

[16] C. Ticknor, e-print arXiv:1104.4277v1.

[17] K. Kanjilal, J. L. Bohn, and D. Blume, Phys. Rev. A 75, 052705 (2007). 
[18] C. Ticknor, R. M. Wilson, and J. L. Bohn, Phys. Rev. Lett. 106, 065301 (2011).

[19] T. Lahaye, C. Menotti, L. Santos, M. Lewenstein, and T. Pfau, Rep. Prog. Phys. 72, 126401 (2009).

[20] T. D. Lee and C. N. Yang, Phys. Rev. 105, 1119 (1957); T. D. Lee, K. Huang, and C. N. Yang, ibid. 106, 1135 (1957).

[21] M. Girardeau, J. Math. Phys. 1, 516 (1960).

[22] I. R. Lapidus, Am. J. Phys. 50, 45 (1982).

[23] S. K. Adhikari, Am. J. Phys. 54, 362 (1986).

[24] M. Schick, Phys. Rev. A 3, 1067 (1971).

[25] E. H. Lieb and J. Yngvason, J. Stat. Phys. 103, 509 (2001).

[26] E. B. Kolomeisky and J. P. Straley, Phys. Rev. B 46, 11749 (1992).

[27] A. Yu. Cherny and A. A. Shanenko, Phys. Rev. E 64, 027105 (2001).

[28] C. Mora and Y. Castin, Phys. Rev. Lett. 102, 180404 (2009).

[29] G. E. Astrakharchik, J. Boronat, J. Casulleras, I. L. Kurbakov, and Yu. E. Lozovik, Phys. Rev. A 79, 051602(R) (2009).
[30] F. Mazzanti, A. Polls, and A. Fabrocini, Phys. Rev. A 71, 033615 (2005).

[31] Handbook of Mathematical Functions with Formulas, Graphs, and Mathematical Tables, edited by M. Abramowitz and I. Stegun (Dover, New York, 1972).

[32] G. E. Astrakharchik, J. Boronat, I. L. Kurbakov, Yu. E. Lozovik, and F. Mazzanti, Phys. Rev. A 81, 013612 (2010).

[33] A. L. Fetter and J. D. Walecka, Quantum Theory of ManyParticle Systems (McGraw-Hill, New York, 1971).

[34] S. Giorgini, J. Boronat, and J. Casulleras, Phys. Rev. A 60, 5129 (1999).

[35] F. Mazzanti, A. Polls, and A. Fabrocini, Phys. Rev. A 67, 063615 (2003).

[36] L. Reatto and G. V. Chester, Phys. Rev. 155, 88 (1967).

[37] E. Krotscheck, in Microscopic Quantum Many-Body Theories and Their Applications, Proceedings of the European Summer School, edited by J. Navarro and A. Polls (Springer, Berlin, 1998).

[38] J. Casulleras and J. Boronat, Phys. Rev. B 52, 3654 (1995). 